\title{
Aerodynamics and Percolation: Unfolding Laminar Separation Bubble on Airfoils
}

\author{
Dominik Traphan, ${ }^{1}$ Tom T. B. Wester, ${ }^{1}$ Gerd Gülker, ${ }^{1}$ Joachim Peinke, ${ }^{1}$ and Pedro G. Lind ${ }^{2,3}$ \\ ${ }^{1}$ ForWind, Institute of Physics, University of Oldenburg, Küpkersweg 70, 26129 Oldenburg, Germany \\ ${ }^{2}$ Institute of Physics, University of Osnabrück, Barbarastrasse 7, 49076 Osnabrück, Germany \\ ${ }^{3}$ Instituto Universitário de Lisboa (ISCTE-IUL), ISTAR-IUL, Avenida das Forças Armadas, \\ 1649-026 Lisboa, Portugal
}

(Received 14 December 2016; revised manuscript received 20 December 2017; published 11 April 2018)

\begin{abstract}
As a fundamental phenomenon of fluid mechanics, recent studies suggested laminar-turbulent transition belonging to the universality class of directed percolation. Here, the onset of a laminar separation bubble on an airfoil is analyzed in terms of the directed percolation model using particle image velocimetry data. Our findings indicate a clear significance of percolation models in a general flow situation beyond fundamental ones. We show that our results are robust against fluctuations of the parameter, namely, the threshold of turbulence intensity, that maps velocimetry data into binary cells (turbulent or laminar). In particular, this percolation approach enables the precise determination of the transition point of the laminar separation bubble, an important problem in aerodynamics.
\end{abstract}

DOI: 10.1103/PhysRevX.8.021015

Subject Areas: Fluid Dynamics, Nonlinear Dynamics, Statistical Physics

\section{INTRODUCTION}

Three decades ago, Pomeau [1] described the flow of a fluid as a collection of oscillators that interact with each other. When observing that "each oscillator if in a turbulent state may either relax spontaneously toward its quiescent state or contaminate its neighbors," he concluded that "this is precisely the definition of the process called "directed percolation' in statistical physics" and therefore raised the possibility of laminar-turbulent transition belonging to the same universality class as directed percolation (DP).

The idea is quite reasonable in the sense that transition from laminar to turbulent flow (hereafter referred to as transition) can be described via the so-called spatiotemporal intermittency [2,3]. Since Pomeau's work, several simulations have supported his conjecture; however, only in the last few years has it been possible to provide experimental evidence [4] because of the novel possibilities of extracting accurate measurements from a turbulent flow with sufficient spatial and temporal resolution. Recent studies approached transition from different angles by means of low-order models [5-7] and sophisticated simulations [8-11], as well as experiments [12-14]. They concordantly indicate nonequilibrium phase transition occurring in basic shear flows, i.e., pipe, channel, and Couette flows. While investigating

Published by the American Physical Society under the terms of the Creative Commons Attribution 4.0 International license. Further distribution of this work must maintain attribution to the author(s) and the published article's title, journal citation, and DOI. the hypothesis of transition belonging to the universality class of directed percolation, those studies treat transition into turbulence as a fundamental phenomenon. Up to now, directed percolation has not been used in flows with a direct aim of helping to solve specific needs in applied sciences that deal with turbulence by extension.

Here, we argue that the applicability of percolation models can be extended from fundamental fluid dynamics to practical aerodynamics relevant for engineering problems and, thus, to a more generally valid concept. More specifically, we provide evidence that directed percolation modeling tools are capable of characterizing the onset of a laminar separation bubble (LSB) on the suction side of an airfoil (see Fig. 1) [15,16]. This is of particular importance for two reasons: First, the aerodynamic functionality of an airfoil depends sensitively on such transitions as the LSB. Second, the precise determination of the onset of transition into the LSB, as well as transition in general, is still an open problem in aerodynamics. So far, there are no methods available that reliably capture the nonlinear nature of a LSB. Because of its known connection with turbulence phenomena, DP is potentially valid for nonlinear flow phenomena encountered in the class of external flows over curved surfaces, where the flow becomes more and more unstable as it is advected downstream. Based on our findings, we discuss how DP properties can also be of use in applications ranging from computational fluid dynamics (CFD), to the design process of airfoils, through the maintenance of rotor blades in wind turbines.

The paper is structured as follows. First, the experimental setup is described in Sec. II. This includes an airfoil 


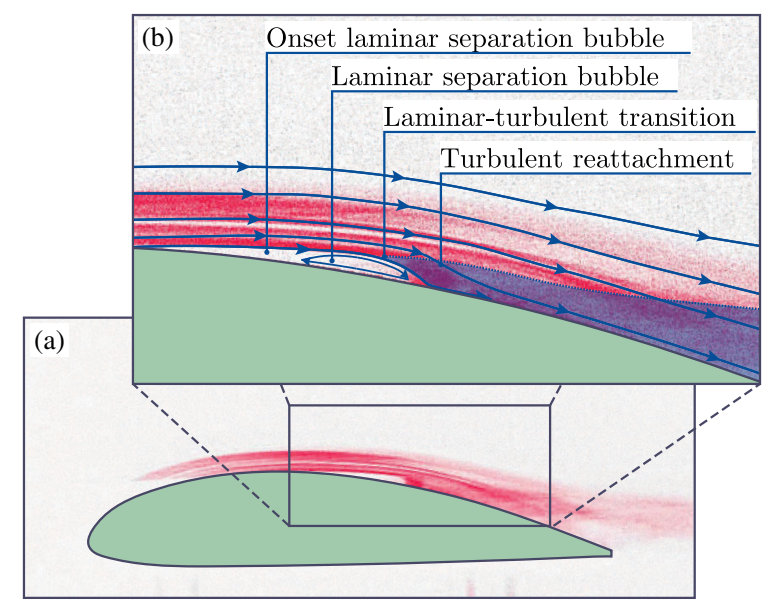

FIG. 1. (a) Photograph of a LSB on airfoil CK220. The laser light sheet illuminates the particle-seeded inflow from the righthand side and perpendicular to the airfoil's surface, resulting in a shadow at the leading edge. The inflow is coming from the left-hand side. The LSB can be identified as a region without smoke between the airfoil's surface and the ambient flow. False colors are used for better visualization. (b) Labeled zoom, emphasizing the flow topology at the LSB. The LSB occurs just behind the airfoil's thickest cross section, where streamlines (in blue) separate from the surface. Further downstream, when the LSB is at its maximum expansion, the laminar shear layer destabilizes, resulting in transition to turbulence. High momentum perpendicular to the airfoil's surface contained in the shear layer enables reattachment and the formation of a turbulent boundary layer.

subjected to laminar inflow and the optical flow visualization method. In Sec. III, a procedure is explained for how to map the measured flow field into binary cells, either laminar or turbulent, which enable the estimation of a critical Reynolds number identifying the onset of the LSB. Characteristic exponents at this critical value are determined in Sec. IV and discussed subsequently in Sec. V. Finally, Sec. VI concludes the paper.

\section{EXPERIMENT}

The phenomenon of the LSB can be qualitatively visualized by means of laser-illuminated smoke photography (see Fig. 1). The seeded flow comes from the left-hand side; it separates just after the airfoil's thickest cross section, destabilizes, and undergoes transition resulting in reattachment of the turbulent boundary layer. The recirculation zone between the airfoil's surface and the shear layer is the one usually addressed as the LSB. In Figs. 1(a) and 1(b), the LSB appears as the smoke-free region above the airfoil. While the transition (taking place within the shear layer between the LSB and free flow) is three dimensional, the LSB's onset happens in a linearly stable laminar flow region whose boundary layer has a thickness that is small compared to the dimensions of the

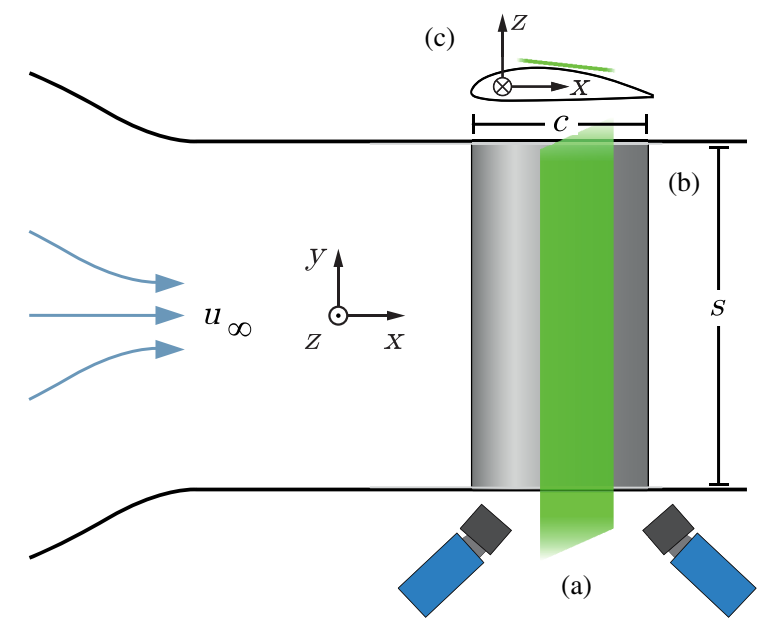

FIG. 2. Experimental setup consisting of (a) a HSPIV system and (b) a CK220 airfoil. A laminar inflow of $u_{\infty}=11 \mathrm{~m} / \mathrm{s}$ approaches the airfoil from the left-hand side, forming a LSB on the suction side. The light sheet (in green) is adjusted (c) tangentially to the surface approximately at the location where the LSB is observed. The coordinate system has its origin at the midspan leading edge and defines the main flow direction as the $x$ direction, the spanwise direction as the $y$ direction, and the normal to wind tunnel wall as the $z$ direction. The chord length and span of the airfoil are denoted $c$ and $s$, respectively.

LSB. Therefore, transition into the LSB can be approximated locally as a quasi-two-dimensional process.

The formation of a LSB is a highly unsteady process that emerges stochastically over a certain region on airfoils. In order to properly investigate this complex and delicate flow topology, it is crucial to use nonintrusive methods featuring high spatiotemporal resolution. Therefore, highspeed stereoscopic particle image velocimetry (HSPIV) is used to visualize a LSB on the suction side of a CK220 airfoil in the wind tunnel. A schematic representation of the experiment is given in Fig. 2. The experimental setup consists of two Phantom Miro M320S high-speed cameras and a Litron LDY303 laser. This enables HSPIV measurements with a recording frequency of 2000 velocity fields per second and a recording length of $T \approx 3 \mathrm{~s}$ at a reduced resolution of $896 \times 792 \mathrm{px}^{2}$. For a measurement region of $\Delta x \times \Delta y=40 \times 40 \mathrm{~mm}^{2}$, where $x$ and $y$ are in a chordwise or spanwise direction, respectively, a spatial resolution of $d x=d y<0.4 \mathrm{~mm}$ is obtained with sufficient accuracy (stereo residue below $0.5 \mathrm{px}$ ). This resolution corresponds to $d x / c<2 \times 10^{-3}$ at a given airfoil dimension of $c \times s=220 \times 250 \mathrm{~mm}^{2}$, where $c$ denotes the airfoil's chord length and $s$ is its span.

As illustrated in Fig. 2, the light sheet is adjusted tangentially to the airfoil's surface approximately at the onset of the LSB at a distance $d z / c<3 \times 10^{-3}$ or $d z=0.5 \mathrm{~mm}$, respectively. Because of the airfoil's curvature, this distance varies slightly, by less than $5 \%$ of the LSB's thickness. The onset of the LSB is thus captured 
properly. At the same time, the measurement region covers the LSB completely.

In the present experiment, the LSB appears at a global Reynolds number of $\mathrm{Re}_{\text {chord }}=160,000$ and an angle of attack of $\alpha=5^{\circ}$. Here, $\operatorname{Re}_{\text {chord }}$ is obtained with respect to the chord length and a free-stream velocity of $u_{\infty}=11 \mathrm{~m} / \mathrm{s}$. In this way, the ambient conditions of the experiment are described in general. Moreover, any transition process is strongly dependent on detailed flow conditions, such as freestream properties. To minimize this impact, the experiments are performed in a wind tunnel with inlet $250 \times 250 \mathrm{~mm}^{2}$, length $2000 \mathrm{~mm}$, and a closed test section with a lowturbulence intensity of TI $<0.004$.

\section{FROM PARTICLE IMAGE VELOCIMETRY DATA TO LAMINAR AND TURBULENT STATES}

Following the objective of this study, the LSB's onset needs to be derived from experimental data. HSPIV results provide instantaneous velocity fields of the whole LSB and the flow upstream as well as downstream of the LSB. Characteristic differences between those three regions are reflected by velocity magnitude $u$ and the fluctuations $u^{\prime}$. Both quantities define the TI. The value of TI is usually derived locally from the whole data set losing its temporal information, whereas directed percolation analysis is based on the time-resolved evolution of the laminar and turbulent regions. Therefore, we define the TI at the time $t$ as the TI computed locally at the position $(x, y)$ from 10 consecutive velocity fields in the following manner:

$$
\begin{aligned}
\langle u(x, y, t)\rangle & \equiv\left\langle u_{t}\right\rangle=\frac{1}{10} \sum_{t^{\prime}=t}^{t+9} u\left(x, y, t^{\prime}\right), \\
\operatorname{TI}(x, y, t) & =\sqrt{\frac{1}{10\left\langle u_{t}\right\rangle^{2}} \sum_{t^{\prime}=t}^{t+9}\left(u\left(x, y, t^{\prime}\right)-\left\langle u_{t}\right\rangle\right)^{2} .}
\end{aligned}
$$

The general mapping procedure into laminar and turbulent states is visualized in Fig. 3. The flow moves along the airfoil's surface (gray) in the $x$ direction, which is chordwise. In the horizontal color-coded plane, the LSB can be clearly identified as a high level of turbulence intensity. Mainly, a velocity magnitude close to zero within the LSB contributes to the sharp rise of TI, while velocity fluctuations increase only slightly. It is important to notice that the term "laminar separation bubble" is misleading at this point. In the context of the present work, the LSB is revealed by a turbulent flow region, whereas it is referred to as laminar in airfoil aerodynamics. This is due to the fact that velocity fluctuations within the LSB are small compared to fluctuations present in a fully turbulent boundary layer. With that in mind, we use a

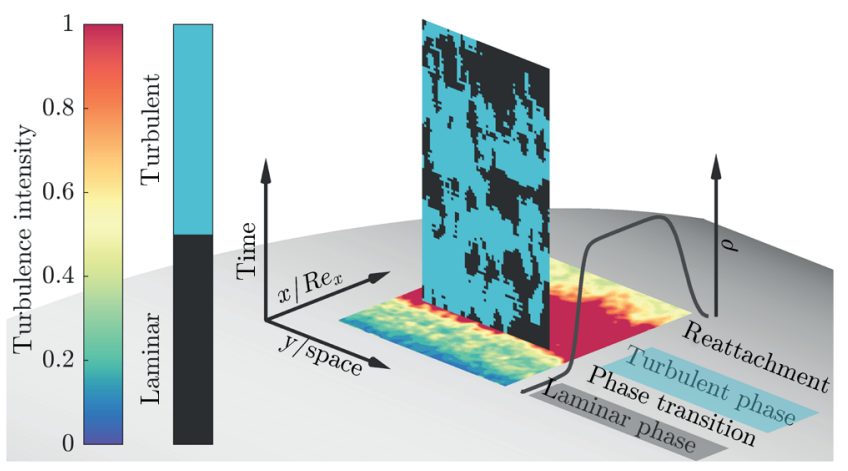

FIG. 3. Illustration of measured and computed quantities. The plane of space and the control parameter $\left(\operatorname{Re}_{x}\right)$ is formed by the airfoil's spanwise and chordwise directions, where $\mathrm{Re}_{x}=$ $\operatorname{Re}\left(x, u_{\infty}\right)$ is based on the distance $x$ from the leading edge and the inflow velocity $u_{\infty}$. Here, $\operatorname{Re}_{x}$ provides a measure for the flow's likelihood to undergo transition from laminar flow into a LSB and, therefore, serves as a control parameter. The presence of a LSB is indicated by means of turbulence intensity (TI) exceeding a certain threshold denoted as turbulent phase. The instantaneous turbulence intensity is derived from 10 temporally consecutive velocity fields at one respective position $\mathrm{Re}_{x}$ in a plane crossing the laminar separation bubble. Transition from laminar flow into the LSB happens after turbulent clusters merge significantly. At this critical point $\operatorname{Re}_{c}$, characteristic turbulent clusters occur, depicted as an evolution in time (vertical plane). Averaging over time at each position $\mathrm{Re}_{x}$, the fraction of turbulent cells, $\rho$, is shown next to the plane of TI. Following $\rho$ in the streamwise direction, transition from the laminar boundary layer flow into the LSB and subsequent reattachment are obvious. The first phase transition represents the LSB's onset, which is important for our work.

threshold value of the turbulence intensity $\mathrm{TI}_{\mathrm{th}}$ to distinguish between laminar and turbulent regions, called clusters. Each measurement point of the complete data set is set to 0 if $\operatorname{TI}(x, y, t)<\mathrm{TI}_{\text {th }}$ (laminar phase) or 1 if $\mathrm{TI}(x, y, t) \geq \mathrm{TI}_{\mathrm{th}}$ (turbulent phase). The turbulent phase corresponds to the LSB, whereas the laminar phase identifies ambient flow.

The onset of the LSB is accurately determined by the evolution of laminar and turbulent clusters. The distribution of clusters is evaluated at each position $x$ along the spatial and temporal dimensions $y$ and $t$, as exemplary illustrated close to the LSB's onset by the bicolored vertical plane in Fig. 3.

\section{TRANSITION INTO LAMINAR SEPARATION BUBBLE}

The flow along the airfoil is more likely to separate from the surface (onset of the LSB) the further downstream from the leading edge it has been evolved because of an increasing receptivity for perturbations. In analogy to well-known transition results from flat plates [17], this is expressed in terms of the local Reynolds number 

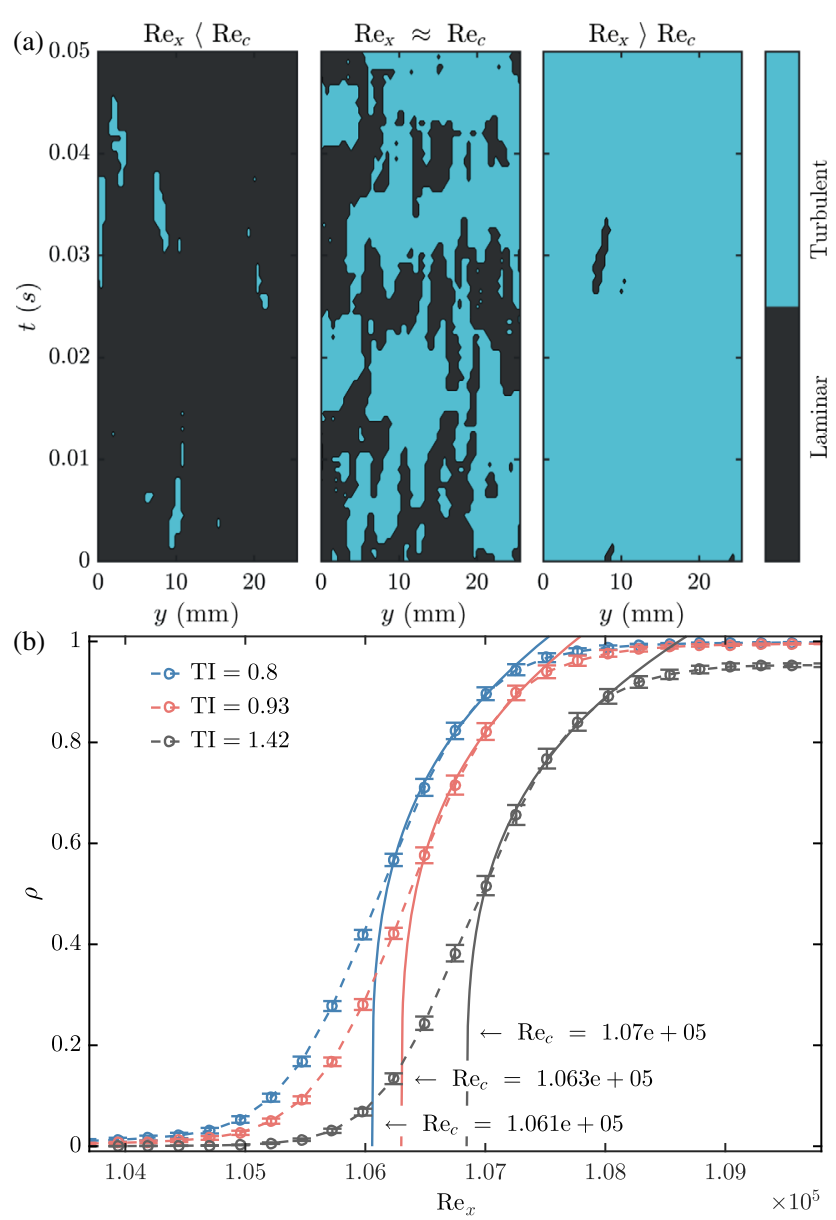

FIG. 4. (a) Three illustrations of cluster distributions in $t$ (time) and $y$ (space) derived for $\mathrm{TI}_{\mathrm{th}}=0.8$ : (Left panel) Reynolds number below the critical value $\mathrm{Re}_{c}$, showing a dominance of laminar clusters. (Right panel) Reynolds number above $\operatorname{Re}_{c}$, where turbulent cells dominate. (Middle panel) Reynolds number at the computed $\operatorname{Re}_{c}$ for which laminar and turbulent clusters span the entire system. (b) Turbulent fraction $\rho$ (circles), as a function of $\operatorname{Re}_{x}$, where $\rho$ is the fraction of cells with a turbulence intensity larger than a threshold $\mathrm{TI}_{\mathrm{th}}$. Results are shown for three different threshold values. The corresponding best fits (solid lines) above the critical point cross the control parameter axis at the critical Reynolds number $\operatorname{Re}_{c}$. Experimental uncertainty of $\rho$ is estimated by the standard error of 10 subsets constituting the total data set.

$\operatorname{Re}(\mathrm{x})=\operatorname{Re}_{x}=x u_{\infty} / \nu_{\text {kin }}$, where $\nu_{\text {kin }}$ denotes the kinematic viscosity. This locally changing $\mathrm{Re}_{x}$ represents the state of the boundary layer and must not be confused with the fixed $\mathrm{Re}_{\text {chord }}$ reflecting the experiment globally. Therefore, $\mathrm{Re}_{x}$ is used as the control parameter in the framework of DP for the present experiment. Notice that one HSPIV snapshot is composed of the control parameter $\operatorname{Re}_{x}$ axis and the spatial dimension $y$ of our percolation model. The time dimension $t$ in this percolation model corresponds to the physical time in the experiment. In this way, the evolution of turbulent clusters is locally captured in a plane held by the spatial dimension $y$ and time $t$ at each $\operatorname{Re}_{x}$.

In accordance with DP, a critical value $\mathrm{Re}_{c}$ of the Reynolds number $\operatorname{Re}_{x}$ is observed, where transition to the LSB takes place. In general, this value is characterized by turbulent clusters merging into one infinitely connected cluster. Figure 4(a) shows three illustrative extracts containing laminar and turbulent clusters in space $y$ and time $t$. While below and above the critical Reynolds number laminar and turbulent clusters, respectively, dominate, the number of laminar and turbulent clusters span the entire system in both directions at $\operatorname{Re}_{c}$.

In the framework of DP, transition from the laminar boundary layer into the LSB is characterized by three critical exponents at $\operatorname{Re}_{c}$. The first exponent, $\beta$, describes the critical behavior of the so-called turbulent fraction,

$$
\rho\left(\operatorname{Re}_{x}\right)=\rho_{0} \varepsilon^{\beta},
$$

as a function of the reduced Reynolds number, $\varepsilon:=$ $\left(\operatorname{Re}_{x}-\operatorname{Re}_{\mathrm{c}}\right) / \mathrm{Re}_{\mathrm{c}}$, and a proportionality factor $\rho_{0}$. The mean fraction is determined from turbulent cells over space $y$ and time $t$ for each value $\operatorname{Re}_{x}$. The other two critical exponents, $\nu_{\perp}$ and $\nu_{\|}$, characterize the diverging correlations of cluster sizes at $\operatorname{Re}_{c}$ in space and time. According to the so-called hyperscaling relation, $\mu=2-\beta / \nu$ [18], the correlation lengths are univocally expressed by the transverse and longitudinal fractal dimensions, $\mu_{\perp}$ and $\mu_{\|}$. These are defined as the exponents that relate the size of laminar clusters and their number, $N\left(L_{\perp}\right) \sim L_{\perp}^{-\mu_{\perp}}$ and $N\left(L_{\|}\right) \sim L_{\|}^{-\mu_{\|}}$, respectively, where $L_{\perp}$ and $L_{\|}$represent the size of laminar clusters measured in the spatial and temporal directions, $y$ and $t$, respectively. By analogy with the DP analysis presented by Refs. [12,13], we estimate, from our measured data, first the critical Reynolds number $\operatorname{Re}_{\mathrm{c}}$ and subsequently the set of critical exponents $\left(\beta, \mu_{\perp}, \mu_{\|}\right)$. The results will be discussed in Sec. V.

The turbulent fraction over local Reynolds number is shown for three descriptive thresholds $\mathrm{TI}_{\mathrm{th}} \in[0.8,0.93$, 1.42] in Fig. 4(b). A transition occurs within the narrow range of values of $\mathrm{Re}_{x}$, where an abrupt increase of the turbulent fraction is observed separating a laminar phase ( $\rho \sim 0)$ from a turbulent phase $(\rho \sim 1)$. For the whole DP analysis, it is important to precisely determine the critical value of $\operatorname{Re}_{c}$.

Note that $\operatorname{Re}_{c}$ and $\beta$ can be obtained simultaneously by a best fit according to Eq. (2). The measurement points used for the best fit are selected in two steps. First, the derivative of the turbulent fraction with respect to the Reynolds number, $\left[(d \rho) /\left(d \operatorname{Re}_{x}\right)\right]$, is computed, as shown in Fig. 5(a). Considering the largest value of the derivative together with the derivative values at the two nearest measured Reynolds numbers [red stars in Fig. 5(a)], a parabola with negative concavity is defined, whose mathematical maximum is taken as an initial estimate of the critical value. While starting the best fit at this estimated location, in step two, only measurement points are taken 

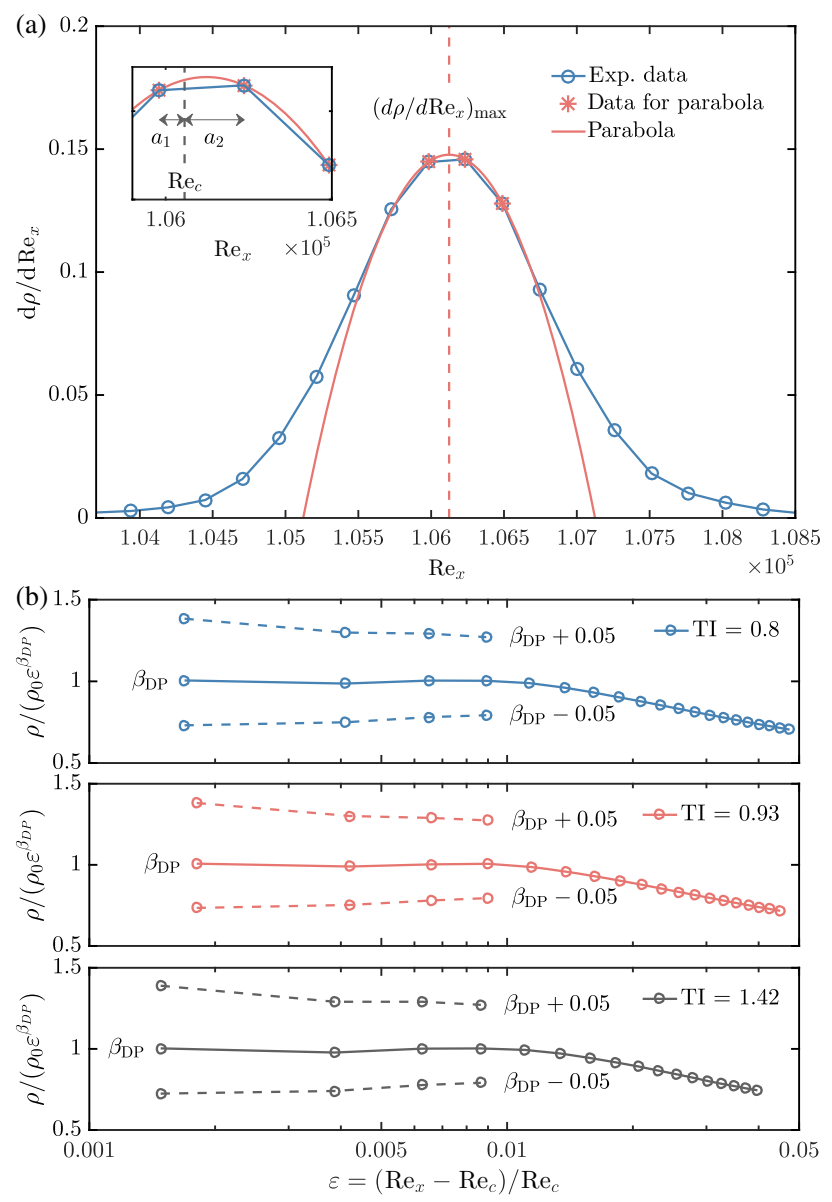

FIG. 5. (a) Illustration of how the critical Reynolds number $\operatorname{Re}_{c}$ is determined: (i) One first adjusts a parabola (red line) around the three highest values (stars) of the derivative of the turbulent fraction $\left[d \rho /\left(d \operatorname{Re}_{\mathrm{x}}\right)\right]$ (circles); (ii) one then performs a best fit, expecting the critical Reynolds number $\operatorname{Re}_{c}$ lying close to the mathematical maximum (red dashed line) of the parabola respecting the spatial resolution of the experiment. Based on this determined interval, $\operatorname{Re}_{c}$ is estimated after Eq. (2). The obtained $\operatorname{Re}_{c}$ is marked in the inset (gray dashed line) along with weights $a_{1}$ and $a_{2}$ used for computation of distributions of laminar cluster sizes at $\operatorname{Re}_{c}$ (see Fig. 6). Here, $\mathrm{TI}_{\text {th }}=0.8$. (b) Compensated plots [after Eq. (3)] for each value of the turbulence intensity threshold: fraction of turbulent cells rescaled to the one for $1+1 \mathrm{D}$ directed percolation predictions as a function of the reduced Reynolds number $\varepsilon$.

into account meeting $0.001<\varepsilon<0.01$, in accordance with the experimental spatial resolution. As shown in Fig. 4(b), this procedure yields values of $\operatorname{Re}_{c}=1.061(9) \times 10^{5}$, $\beta=0.28(4)$ for $\mathrm{TI}_{\mathrm{th}}=0.8, \mathrm{Re}_{c}=1.063(6) \times 10^{5}, \beta=$ $0.28(0.05)$ for $\mathrm{TI}_{\mathrm{th}}=0.93$, and $\operatorname{Re}_{c}=1.07(1) \times 10^{5}$, $\beta=0.28(5)$ for $\mathrm{TI}_{\mathrm{th}}=1.42$. In comparison, the predicted theoretical value of $1+1 \mathrm{D}$ directed percolation is $\beta_{D P}=$ 0.276 .

In order to evaluate the validity of the determined characteristic values $\operatorname{Re}_{c}$ and $\beta$, the rescaled turbulent fraction $\tilde{\rho}$, defined as

$$
\tilde{\rho}\left(\operatorname{Re}_{x}\right)=\frac{\rho\left(\operatorname{Re}_{x}\right)}{\rho_{0} \varepsilon^{\beta_{D P}}},
$$

is shown in Fig. 5(b) as a function of the reduced Reynolds number using the theoretical value of $\beta_{D P}$. In this representation, scaling is readily apparent as a horizontal line equal to unity. Dashed lines also show the rescaled fraction obtained for slight deviations from the exponent, $\beta_{D P} \pm 0.05$.

To estimate the other two critical exponents describing the fractal dimensions, the distributions of the sizes of the laminar clusters at the critical Reynolds number have to be known. Based on our finite experimental resolution, we have no direct access to these critical distributions. To overcome this problem, we take the corresponding distributions at the two Reynolds numbers close to $\operatorname{Re}_{c}$ and sum them up in a weighted manner using the normalized inverse of their distances $a_{1}$ and $a_{2}$ to $\operatorname{Re}_{c}$ [cf. inset of Fig. 5(a)].

While the procedure of estimating the critical size distributions is not standard, it results in robust estimates of spatial critical exponents $\mu_{\perp}$. The distributions of cluster sizes in space and time at the critical Reynolds number are shown in Figs. 6(a) and 6(b), respectively. The evaluations are performed with the same three threshold values $\mathrm{TI}_{\mathrm{th}}$ as used previously. Each best fit for the spatial fractal dimension (smallest three cluster sizes are disregarded) yields $\mu_{\perp}=1.75(9)$ and compares well with the theoretical value predicted by directed percolation, $\mu_{\perp, D P}=1.748$. For the temporal direction, the results are not as conclusive as for the spatial direction. Seemingly, the cluster distribution tends towards exponential behavior and, because of the continuous change in curvature, no exponent can be fitted to our data. The theoretical exponent $\mu_{\|, D P}=1.84$ is shown in Fig. 6(b) with a dashed line for comparison. Here, we note that the temporal analysis is more affected by experimental perturbations, as we work out in the Appendix.

As a last step in our data analysis, we investigate the effect of the choice of threshold values $\mathrm{TI}_{\mathrm{th}}$ for this transition. Beyond the three threshold values considered up to now, Fig. 7 shows the results for two of the three critical exponents with $95 \%$ confidence intervals for 200 values of $\mathrm{TI}_{\mathrm{th}}$ covering a range of $0.5<$ $\mathrm{TI}_{\mathrm{th}}<2.5$. The theoretically predicted values of the critical exponents are shown by horizontal dashed lines. It is evident that the values of the $\beta$ and $\mu_{\perp}$ exponents are robust against the change of the turbulence intensity threshold for $\mathrm{TI}_{\mathrm{th}}>0.64$.

A variation of a factor of 5 in turbulence intensity threshold implies an increase of less than $2 \%$ of the critical Reynolds number, while the corresponding uncertainty is on the order of $1 \%$. The monotonic trend is a natural consequence of mapping continuous HSPIV velocity data into binary states using increasing threshold values $\mathrm{TI}_{\mathrm{th}}$. As it is ubiquitous in phase transitions, the value of the control 

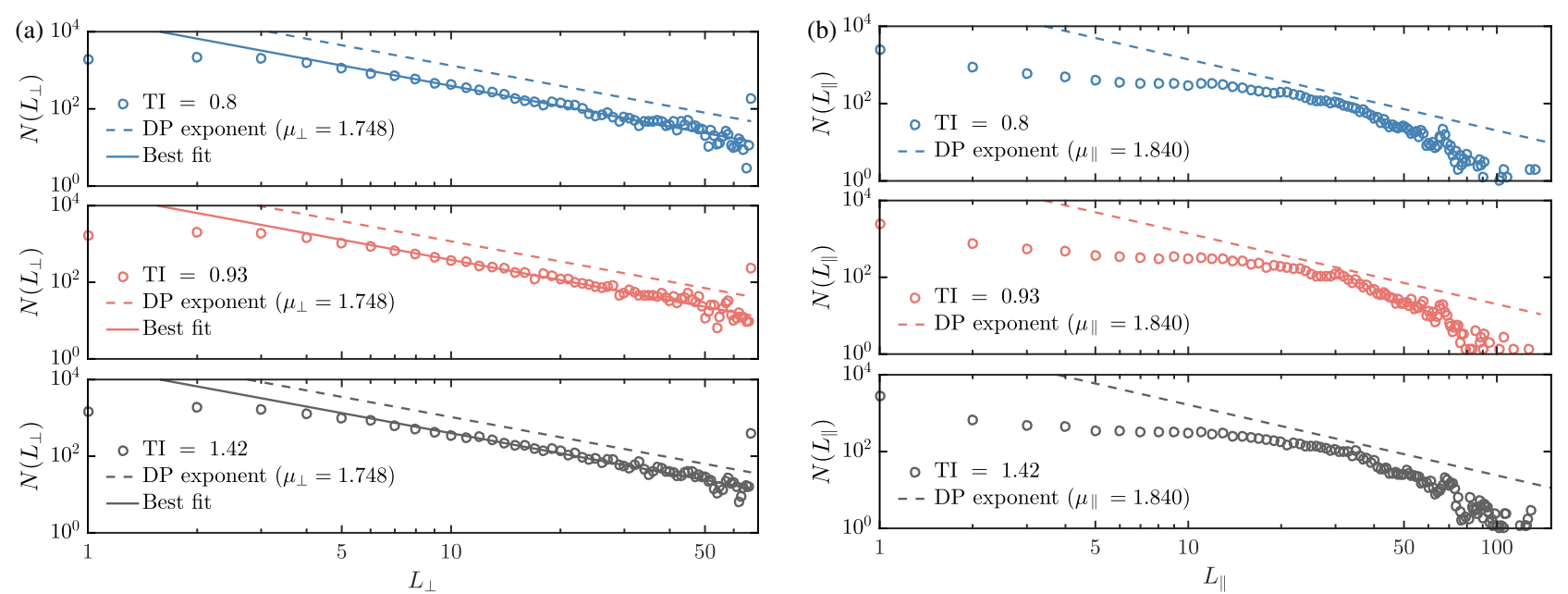

FIG. 6. (a) Size distribution of laminar clusters in space at the estimated critical Reynolds number, i.e., consecutive laminar states when sweeping in the $y$ direction at a given time $t$, for three illustrative values of turbulence intensity thresholds and (b) their corresponding size distribution of laminar clusters in time, i.e., consecutive laminar states when sweeping in the time $t$ while keeping $y$ constant. Solid lines show the theoretical distribution predicted by the directed percolation model at the transition. For comparative purposes, the turbulence intensity threshold values are the same as the ones in Fig. 4(b), where the critical value for each TI is given. Cluster lengths are shown in multiples of the spatial and temporal resolution, respectively.

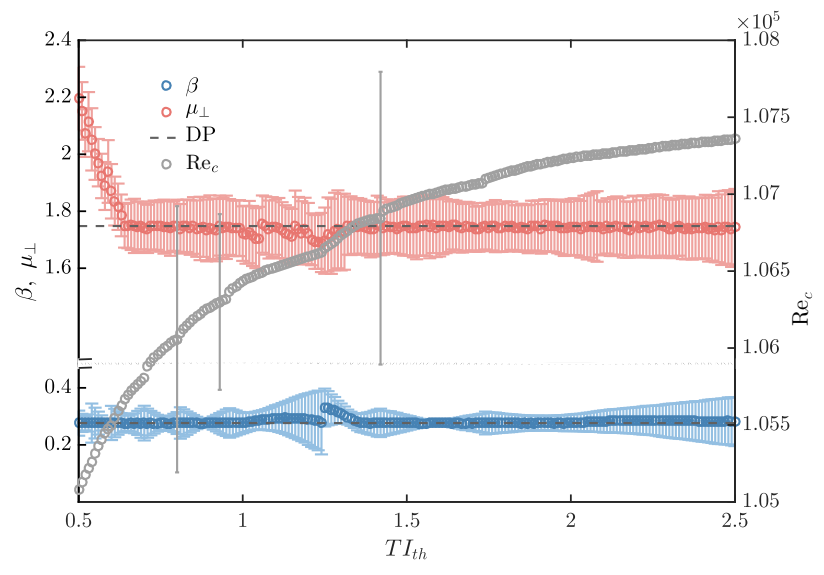

FIG. 7. Systematic analysis of the critical exponents sensitivity to the threshold of turbulence intensity imposed in the directed percolation model: the exponent $\beta$ and the transverse fractal dimension $\mu_{\perp}$. The dashed lines indicate the theoretical value of the exponents in directed percolation. For a turbulence intensity $\mathrm{TI}_{\mathrm{th}}>0.64$, the critical exponents of directed percolation are obtained within numerical errors (see text). Gray symbols show the dependence of the critical Reynolds number on the threshold. For the sake of clarity, only three illustrative error bars of $\mathrm{Re}_{c}$ are shown.

parameter at which a phase transition occurs is model dependent. For claiming universal behavior at the phase transition, it is important to observe critical exponents that do not depend on these model details. All these features are observed in our investigations (Fig. 7). We thus conclude that the critical Reynolds number marking the onset of the LSB depends only slightly on $\mathrm{TI}_{\text {th }}$ without contradicting DP.

\section{DISCUSSION}

The main aspect of our work is to show evidence for the concept of DP being of use for understanding transition phenomena in aerodynamics, in particular, the onset of a LSB on an airfoil. This evidence is shown by means of estimated critical exponents. However, as it is known, this estimation is very sensitive to the computation or measurement of the critical point, or here, the critical Reynolds number. To show the quality of our estimate, we present compensated plots in Fig. 5(b). For the critical exponents of the turbulent fraction $(\beta)$ as well as the transverse fractal dimension $\left(\mu_{\perp}\right)$, Figs. 5(b), 6(a), and 7 show accordance with the predicted values of $1+1 \mathrm{D}$ directed percolation, even if one varies the confidence intervals for the error estimation. The results of the longitudinal fractal dimension $\left(\mu_{\|}\right)$are not so conclusive. Tending towards exponential behavior, distribution of laminar clusters actually contradicts $1+1 \mathrm{D}$ directed percolation [see Fig. 6(b)]. Since cluster distributions in time are known to be particularly more difficult to estimate as they seem to suffer more from finite size effects and experimental uncertainty, our findings open the door for future experimental sets: Minimizing the uncertainty may uncover a critical behavior that is not resolved in our experiment, or it may provide deeper evidence for the occurrence of a phenomenon other than critical phase transition, e.g., a continuous bifurcation.

The scaling ranges obtained for our systems are not much larger than one decade. However, in contrast to numerical investigations, we are limited by our experimental setup. Neither the spatial dynamic range of HSPIV nor 
the size of the wind tunnel can easily be changed. Also taking into account that estimation of power laws may lead to biased estimation and pitfalls [19], our experimental results indicate, to the best of our knowledge, astonishingly consistent results with the $1+1 \mathrm{D}$ directed percolation. We also see that the quality of our results is comparable with that of other groups, like the work on directed percolation in a channel flow [13]. Other experimental studies on DP $[11,20]$ suffer from similar shortcomings, such as the continuous onset of turbulence and truncated scaling ranges. By establishing an adapted DP model, we are able to trace these shortcomings back to typical experimental constraints, such as noisy control parameters and experimental coarse graining. We introduce this DP model in the Appendix and show that these findings explain the experimental deviations from theory and thus provide stronger grounding for the potential importance of the DP framework in LSB events and other transition phenomena in aerodynamics.

Based on the findings in this paper, it is now possible to introduce an alternative procedure to determine the onset of the LSB. Under the assumption that 1+1D DP holds true, the critical Reynolds number can be determined following the compensated representation shown in Fig. 5(b) but now fixing $\beta_{D P}$ to its theoretical value and varying a reference value $\operatorname{Re}_{c^{\prime}}$, which is deviated from the critical value,

$$
\hat{\rho}\left(\operatorname{Re}_{x}, \operatorname{Re}_{c^{\prime}}\right)=\frac{\rho}{\varepsilon^{\beta_{D P}}} .
$$

For a variety of reference Reynolds numbers, $\hat{\rho}$ is shown as a function of $\varepsilon$ and $\operatorname{Re}_{c^{\prime}}$ in Fig. 8(a). Here, we see that by increasing (decreasing) $\operatorname{Re}_{c^{\prime}}$, the slope $\hat{\rho}^{\prime}=\partial \hat{\rho} / \partial \varepsilon$ becomes
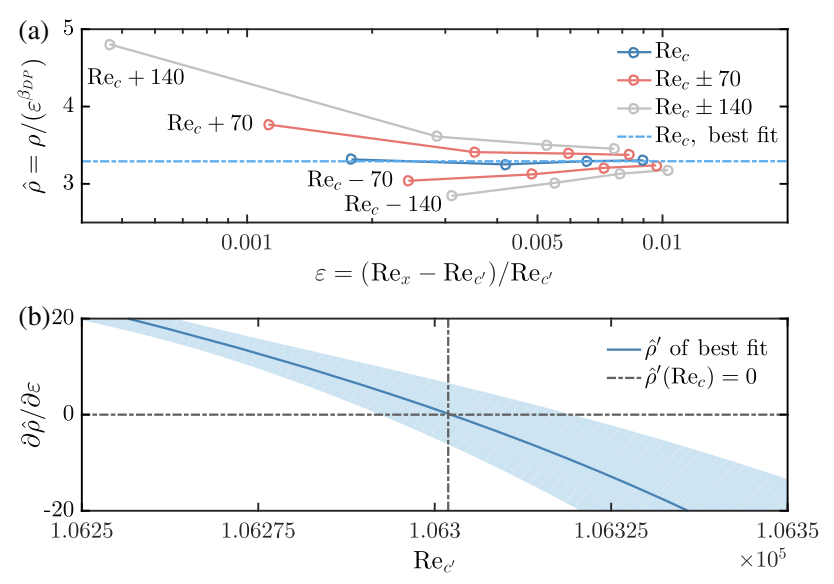

FIG. 8. (a) Rescaled turbulent fraction $\hat{\rho}$ as a function of $\varepsilon$ for a variety of possible critical Reynolds numbers, $\operatorname{Re}_{c^{\prime}} \in$ $\left[\operatorname{Re}_{\mathrm{c}}, \operatorname{Re}_{\mathrm{c}} \pm 75, \operatorname{Re}_{\mathrm{c}} \pm 150\right]$. Where $\hat{\rho}$ becomes constant, $\operatorname{Re}_{c}$ can be estimated best. (b) Derivative of $\hat{\rho}$ as a function of $\operatorname{Re}_{c^{\prime}}$ including standard errors. The critical Reynolds number is determined by the zero crossing of $\hat{\rho}^{\prime}$. The uncertainty of $\operatorname{Re}_{c}$ is estimated by propagation of errors. Here, $\mathrm{TI}_{\mathrm{th}}=0.93$. negative (positive). By analogy with Fig. 5(b), the horizontal line in $\hat{\rho}$ represents the case where DP properties are found. Thus, from the zero crossing of $\hat{\rho}^{\prime}$, the best estimation $\operatorname{Re}_{c}$ is obtained. In comparison to the estimation of the critical Reynolds number by fitting a power law about the maximum derivative of $\rho$ [see Figs. 4(b) and 5(a)], the uncertainty in $\operatorname{Re}_{c}$ decreases by 2 orders of magnitude from $\operatorname{Re}_{c}=1.063(6) \times$ $10^{5}$ to $\operatorname{Re}_{c}=1.06302(2) \times 10^{5}$ for $\mathrm{TI}_{\mathrm{th}}=0.93$. This shows that the concept of DP enables us to determine the critical Reynolds number for the LSB with very high precision. Since such high-precision concepts are very rare in fluid mechanical research, the procedure introduced may serve as a new benchmark.

\section{CONCLUSION}

This work presents the first experimental evidence that percolation models are of practical relevance for the flow over the suction side of an airfoil. Our work has been inspired by recent achievements in fundamental turbulence research that link the onset of turbulence to directed percolation phase transition. In comparison to the flow situations investigated up to now, the flow over an airfoil changes its Reynolds number along with its stability while evolving downstream.

Applying a bond directed percolation model to characterize transition from a laminar boundary layer into a LSB on an airfoil, one obtains values for the critical exponents consistent with those in $1+1 \mathrm{D}$ directed percolation. The physical implication of this universality class indicates that the boundary layer at the LSB's onset is slender compared to the dimension of the LSB; thus, flow instabilities cannot spread perpendicularly to the surface. As an important aspect for practical applications, with the assumption of a $1+1 \mathrm{D}$ directed percolation, a new method is introduced to determine the transition point into the LSB with very high precision of better than 10 in Reynolds numbers. This is for fixed TI less than 1 per mill or for all TI less than $1 \%$ of the chord length. For our profile, the precision exceeds the optical resolution of $0.4 \mathrm{~mm}$.

Since instabilities like the LSB have an essential impact on the performance of airfoils, it is of great importance to know how and where they emerge. From the findings of this work, new directions for future applications and investigation are now open. First, in CFD, several frameworks need a model that delivers the location of transition [21] and, thus, the LSB's onset. Since the directed percolation framework is now shown to be able to retrieve a consistent determination where the LSB onset is located, it can be used to validate current transition models. Additionally, the temporal evolution of the LSB's onset parametrized by DP might be of use for unsteady low-order models [22] which often couple details of the boundary layer flow with CFD approaches used for the ambient flow [23,24].

Second, in many applications, transition to turbulence is a phenomenon that needs to be controlled properly, also 
when taking place within a LSB. For instance, vortex generators are applied to rotor blades of wind turbines close to the location of a LSB in order to avoid aerodynamic instabilities that cause high-fatigue loads. Nowadays, this is done based on efficient engineering models used in the design process of a wind turbine. As revealed by different studies [25,26], these models cannot account for nonlinear flow behavior that inherently governs the emergence of LSBs. Particularly, when a LSB emerges very close to the natural onset of laminar-turbulent transition, models fail to correctly predict both phenomena. The more reliably such situations can be identified and characterized, the better vortex generators can be applied to rotor blades, resulting in more efficient wind-turbine operation along with reduction of destructive aerodynamic loads.

Third, in the wind-tunnel experiment investigated here, we consider an airfoil subjected to a constant ambient inflow. In reality, airfoils of a plane or a wind turbine are subjected to nonstationary inflows. Assuming that the nonstationarity of such flow occurs at a smaller time scale than the time scale needed for the LSB onset to take place as a percolation transition, the concept of directed percolation has the potential to derive a model for the (nonstationary) dynamics of the LSB's onset in time.

All in all, the results of the present study indicate a more comprehensive significance of percolation models in fluid mechanics beyond fundamental laminar-turbulent transition phenomena.

\section{ACKNOWLEDGMENTS}

The authors thank Philipp Maaß, Iván Hérraez, Matthias Schramm, Matthias Wächter, and Bruno Eckhardt for comments, and acknowledge Jarek Puczylowski for technical advice. Financial support for this research was provided by the Federal Ministry for Economic Affairs and Energy of Germany in the DFWind project under Grant No. 0325936C and the German Research Foundation in the DFG PAK 780 project.

\section{APPENDIX: DIRECTED PERCOLATION WITH FLUCTUATING CONTROL PARAMETERS}

The results of the present study basically reveal three main deviations from standard DP, which are also present in previous experiments [12,13]: Turbulent clusters appear unconnected and dense, phase transition is rounded off, and scaling ranges are truncated. While the latter two observations, to some degree, are affected by well-known finite size effects, in this appendix, we show that all three apparent deviations from standard DP reflect two important experimental features, namely, limited measurement resolution and a "fluctuating control parameter." The limited measurement resolution is related to the apparently unconnected clusters, while the fluctuating control parameter $\mathrm{Re}_{x}$, which is caused by noisy inflow conditions in the experiment, reflects the rounded-off phase transition. Both experimental features affect the distribution of laminar clusters.

We therefore introduce a modified DP model that incorporates these experimental features, retrieves results resembling laminar-turbulent transition in shear flows [5], and, in particular, presents the same deviations observed in our experimental HSPIV data.

The standard DP model is a two-parameter model discretized in space and time with two different (fixed) probabilities - one for turbulence prevalence in consecutive time steps, $p$, and another for turbulent spreading to neighboring cells, $r$. The flow field is represented by a square lattice with $N \times N$ binary cells, which can be either turbulent (state 1) or laminar (state 0). The binary flow fields are initialized with a fully occupied turbulent system at $t=0$. Turbulent decay or spreading is then investigated, depending on the chosen values for $p$ and $r$. Reference [5] provides all additional details on the standard DP model.

The modified DP model extends the standard DP model in two ways:

(I) Turbulent and laminar cells are coarse grained, merging into larger cells that take binary states depending on the majority of smaller cells within. This extension accounts for natural constraints when capturing a continuous flow by discrete measurement techniques. In the case of HSPIV, the implied averaging imposes a spatiotemporal low-pass filter on continuous flow data.

(II) The turbulence spreading probability $r$ is no longer fixed but varies according to a normal distribution of fixed (small) standard deviation $\sigma_{r}$ and an average value $\langle r\rangle$. The average value plays the role of the new control parameter. A value of $\sigma_{r} \gtrsim 0$ accounts for experimental uncertainties in the

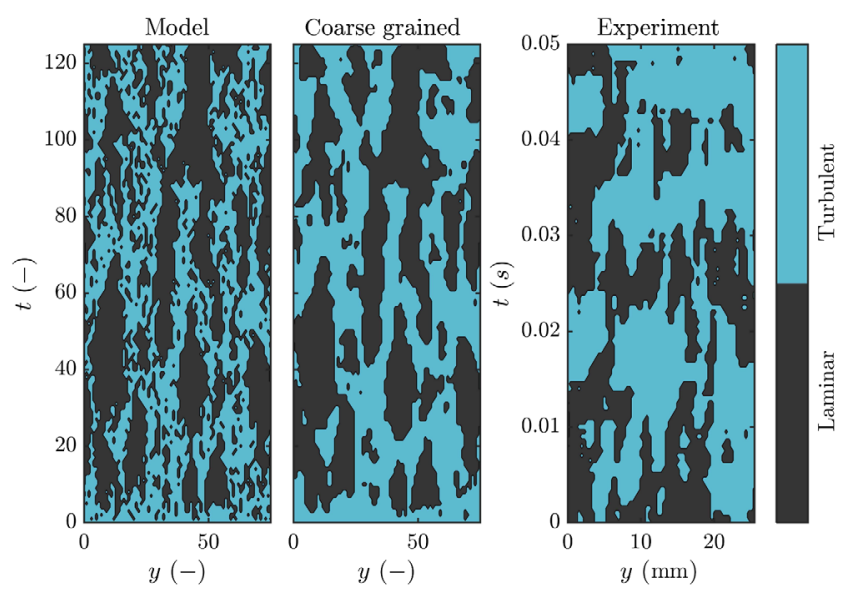

FIG. 9. Illustrations of cluster distributions in time and space at criticality. In the right diagram, a typical snapshot obtained from the experimental HSPIV data is depicted. In the left diagram, the (detailed) result from a DP simulation is compared with the corresponding coarse-grained visualization. 


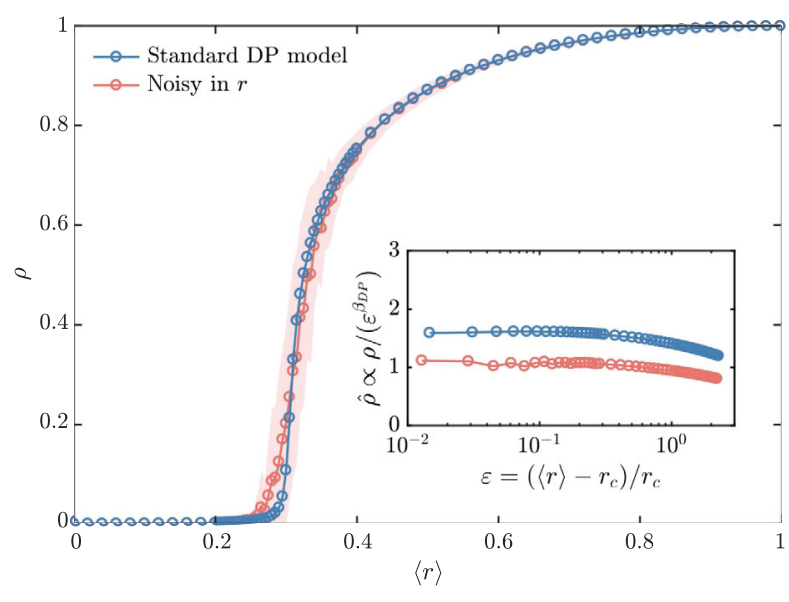

FIG. 10. Turbulent fraction for the baseline DP model (in blue) according to Ref. [5], compared with a modified model (in red) that allows a noisy parameter $r$, which changes in time according to a standard normal distribution with a standard deviation of $\sigma \approx 0.02$ and a fixed average $\langle r\rangle$. This average value plays the role of the "new" control parameter. In the inset, the respective compensated representation indicates a first-order phase transition for both cases. Please note that the critical point is adjusted to each model.

inflow, resulting in a deviation at the critical value $r_{c} \sim\langle r\rangle_{c}$. In the particular case with $\sigma_{r}=0$, the modified model reduces to the standard DP model.

To illustrate the first extension (I), we simulate a flow field on a lattice with $N=5000$, exploiting accurate determination of the critical point as given in Ref. [5]. Subsequent analysis is based on fixed turbulent propagation of $p=0.7$, yielding a critical turbulent spreading of $r_{c} \approx 0.3069$. An example of a simulated cluster distribution in time and space is depicted in Fig. 9 (left diagram), along with the corresponding coarse-grained visualization using a $3 \times 3$ kernel (middle diagram), and compared to typical experimental data (right diagram). Merging simulation data into a coarse-grained representation of larger cells thus yields results qualitatively similar to our experimental space-time plots. In other words, connected clusters in our experimental flow field can appear unconnected and dense because of coarse graining that results from the HSPIV measurement procedure.

To illustrate the second extension (II), we choose a value centered at a fixed average, $\langle r\rangle$, with a normal probability density function, with $\sigma=0.02$ for the fluctuation. In Fig. 10, the turbulent fraction $\rho$ derived from the standard model is compared with the modified DP model. Unlike perfect systems underlying DP (blue curve), the transition is smeared (red curve), a feature also observed in our experiment [cf. Fig. 4(b)]. Although the two curves reveal different behavior about the critical value, the inset of Fig. 10 shows that critical behavior can be recovered in the compensated representation.

Finally, one should also notice that experimental coarse graining, as well as the noisy parameter $r$, both affect the distributions of laminar clusters derived from our DP model for the three cases, as depicted in Fig. 11. Based on a snapshot of the (standard) DP simulation $(N=5000)$, both the transversal and the longitudinal gap distributions show scaling behavior over at least two decades. If the noisy parameter is introduced, the scaling range is significantly restricted in the long clusters. Note that longitudinal clusters are more strongly affected by such noise, as we observe in our experimental data depicted in Fig. 6. Once
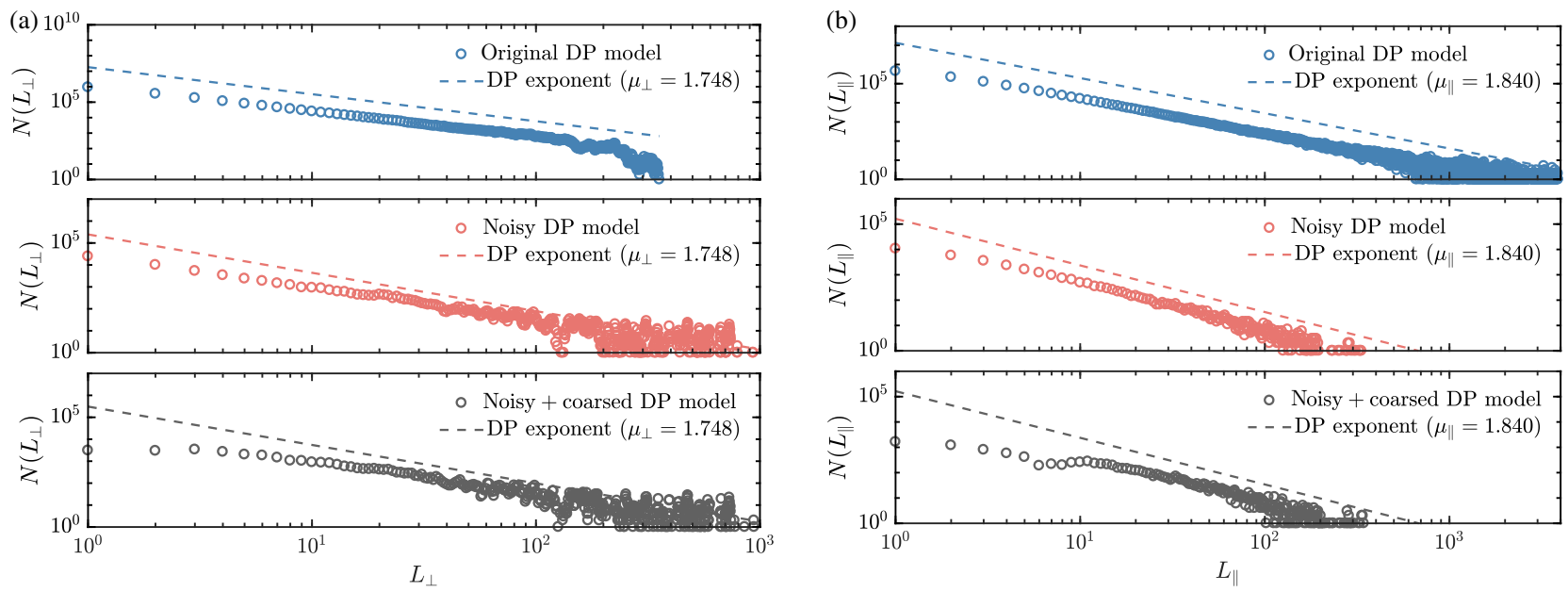

FIG. 11. (a) Size distribution of laminar clusters in space at the estimated critical value (i.e., turbulent spreading) for one illustrative binary flow field generated by applying the baseline DP model (in blue) as presented in Ref. [5]. The baseline model is then modified according to experimental constraints, allowing a fluctuating control parameter (in red) and considering metrological coarse graining (in gray). (b) The corresponding size distribution of laminar clusters in time, depicted analogously. Dashed lines show the theoretical distribution predicted by the directed percolation model at the transition. Cluster lengths are shown in multiples of the discretization used in the simulation. 
coarse graining is added to noise, the number of short clusters is reduced below the edge of the kernel. Particularly, the distribution of longitudinal laminar clusters is affected, showing an apparently exponential behavior.

[1] Y. Pomeau, Front Motion, Metastability and Subcritical Bifurcations in Hydrodynamics, Physica D (Amsterdam) 23, 3 (1986).

[2] H. Chaté and P. Manneville, Transition to Turbulence via Spatiotemporal Intermittency, Phys. Rev. Lett. 58, 112 (1987).

[3] P. Rupp, R. Richter, and I. Rehberg, Critical Exponents of Directed Percolation Measured in Spatiotemporal Intermittency, Phys. Rev. E 67, 036209 (2003).

[4] Y. Pomeau, The Long and Winding Road, Nat. Phys. 12, 198 (2016).

[5] K. T. Allhoff and B. Eckhardt, Directed Percolation Model for Turbulence Transition in Shear Flows, Fluid Dyn. Res. 44, 031201 (2012).

[6] M. Sipos and N. Goldenfeld, Directed Percolation Describes Lifetime and Growth of Turbulent Puffs and Slugs, Phys. Rev. E 84, 035304(R) (2011).

[7] D. Barkley, Simplifying the Complexity of Pipe Flow, Phys. Rev. E 84, 016309 (2011).

[8] H.-Y. Shih, T.-L. Hsieh, and N. Goldenfeld, Ecological Collapse and the Emergence of Travelling Waves at the Onset of Shear Turbulence, Nat. Phys. 12, 245 (2016).

[9] T. Kreilos, B. Eckhardt, and T. M. Schneider, Increasing Lifetimes and the Growing Saddles of Shear Flow Turbulence, Phys. Rev. Lett. 112, 044503 (2014).

[10] M. Avila and B. Hof, Nature of Laminar-Turbulence Intermittency in Shear Flows, Phys. Rev. E 87, 063012 (2013).

[11] A. Doostmohammadi, T. N. Shendruk, K. Thijssen, and J. M. Yeomans, Onset of Meso-scale Turbulence in Active Nematics, Nat. Commun. 8, 15326 (2017).

[12] G. Lemoult, L. Shi, K. Avila, S. V. Jalikop, M. Avila, and B. Hof, Directed Percolation Phase Transition to Sustained Turbulence in Couette Flow, Nat. Phys. 12, 254 (2016).

[13] M. Sano and K. Tamai, A Universal Transition to Turbulence in Channel Flow, Nat. Phys. 12, 249 (2016).
[14] D. Barkley, B. Song, V. Mukund, G. Lemoult, M. Avila, and B. Hof, The Rise of Fully Turbulent Flow, Nature (London) 526, 550 (2015).

[15] H. P. Horton, Ph.D. thesis, Queen Mary, University of London, 1968.

[16] M. O'meara and T. J. Mueller, Laminar Separation Bubble Characteristics on an Airfoil at Low Reynolds Numbers, AIAA J. 25, 1033 (1987).

[17] H. Schlichting and K. Gersten, Boundary-Layer Theory (Springer Science \& Business Media, New York, 2003).

[18] K. A. Takeuchi, M. Kuroda, H. Chaté, and M. Sano, Directed Percolation Criticality in Turbulent Liquid Crystals, Phys. Rev. Lett. 99, 234503 (2007).

[19] A. Clauset, C. R. Shalizi, and M. E. J. Newman, Power-Law Distributions in Empirical Data, SIAM Rev. 51, 661 (2009).

[20] M. Chantry, L. S. Tuckerman, and D. Barkley, Universal Continuous Transition to Turbulence in a Planar Shear Flow, J. Fluid Mech. 824, R1 (2017).

[21] P. R. Spalart and S. R. Allmaras, A One-Equation Turbulence Model for Aerodynamic Flows, in 30th Aerospace Sciences Meeting and Exhibit (American Institute of Aeronautics and Astronautics, USA, 1992), p. 439, https://doi .org/10.2514/6.1992-439.

[22] F. R. Menter, P. E. Smirnov, T. Liu, and R. Avancha, A OneEquation Local Correlation-based Transition Model, Flow Turbul. Combust. 95, 583 (2015).

[23] P. R. Spalart, W. H. Jou, M. Strelets, S. R. Allmaras et al., Comments on the Feasibility of LES for Wings, and on a Hybrid RANS/LES Approach, Adv. DNS/LES 1, 4 (1997).

[24] M. Strelets, Detached Eddy Simulation of Massively Separated Flows, in 39th Aerospace Sciences Meeting and Exhibit (American Institute of Aeronautics and Astronautics, USA, 2001), p. 879, https://doi.org/10.2514/6 .2001-879.

[25] C. Bak, H. A. Madsen, P. Fuglsang, and F. Rasmussen, Observations and Hypothesis of Double Stall, Wind Energy 2, 195 (1999).

[26] J. W. Ward, The Behaviour and Effects of Laminar Separation Bubbles on Aerofoils in Incompressible Flow, Aeronautical J. 67, 783 (1963). 